\title{
THE CHEMICAL COMPOSITION OF LATE TYPE STARS
}

\author{
A. A. BOYARCHUK \\ Crimean Astrophysical Observatory, USSR Academy of Sciences, Crimea, U.S.S.R.
}

\begin{abstract}
A discussion is given of the chemical composition of the atmospheres of various types of late type peculiar stars and their compositions are compared with those of normal $\mathrm{K}$ and $\mathbf{M}$ stars. The groups considered include $\mathrm{CN}, \mathrm{CH}, \mathrm{Ba}, \mathrm{S}, \mathrm{C}, \mathrm{R} \mathrm{CrB}$ and $\mathrm{HdC}$ stars.
\end{abstract}

The chemical compositions of the atmospheres of many stars have been investigated at the present time. Although most of stars investigated have a chemical composition of their atmospheres very close to that of the Sun there are many stars which show pecularities of the chemical composition. The pecularities may arise by two reasons:

(i) The differences in the chemical composition of pre-stellar matter.

(ii) Changing of the chemical composition of stellar surfaces during stellar evolution.

Many astronomers have investigated the chemical composition of stars belonging to the different stellar groups which have different ages. It was established that the older stars have the lower abundances of metals in their atmospheres. Most significant variation of the chemical composition took place during the first $2 \times 10^{8}$ years of life of the Galaxy, when the ratio $[\mathrm{Fe} / \mathrm{H}]$ increased from -2 to 0 . (Here and later $[\mathrm{Fe} / \mathrm{H}]$ means the value $\lg [N(\mathrm{Fe}) / N(\mathrm{H})]_{\text {star }}-\lg [N(\mathrm{Fe}) / N(\mathrm{H})]_{\text {sun. }}$.) After that variations up to factors of \pm 2 from the solar metal abundance occurred (Eggen and Sandage, 1969). The reason of that variation is still unknown (Arp, 1962).

Another important aspect of a problem of chemical compositions of a pre-stellar matter is the relative abundances of individual metals. Pagel (1970) has shown that the $s$-process elements are less abundant in the stars which have smaller values of $[\mathrm{Fe} / \mathrm{H}]$. Especially it is true for the barium.

Thus the general tendency of aging effect of chemical composition is the increasing of the values of $[\mathrm{Met} / \mathrm{H}]$ and of $[$ heavy met/Fe] during time.

However, from point of view of the present symposium the variations of chemical compositions of stellar surfaces which occurred during stellar evolution are much more interesting. We excluded from consideration the main sequence stars because they are not a topic of this symposium. We concentrate our attention on the late-type giants because the rather detailed observations are known for these stars.

We will consider the different groups of stars which show remarkable peculiarities in their spectra and which are located on the giant branch on the spectrum-luminosity diagram (Figure 1). These groups are following:

(1) Normal $\mathrm{K}-\mathrm{M}$ giants.

(2) $\mathrm{CN}$-stars, which have strong $\mathrm{CN}$ bands in their spectra.

(3) $\mathrm{CH}$-stars, which have strong $\mathrm{CH}$-bands in their spectra.

(4) Barium stars, which show abnormal strong Ball lines. 


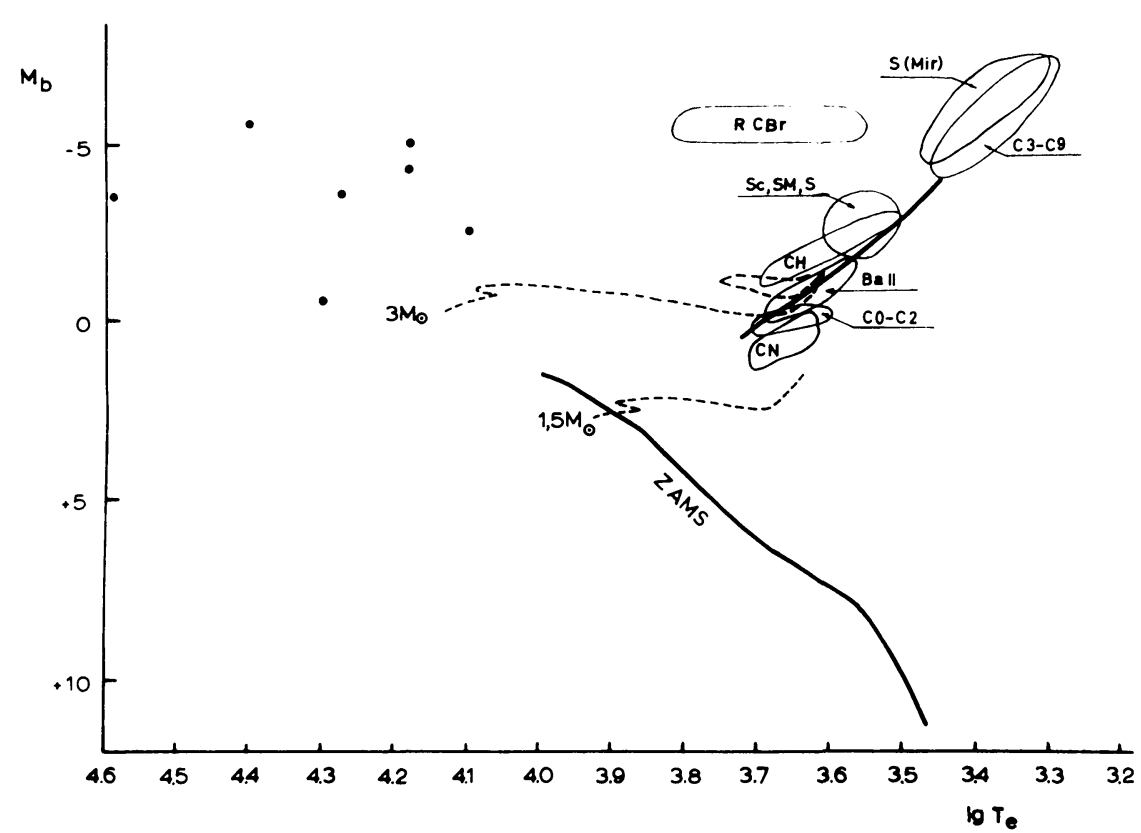

Fig. 1. The location of the different groups of stars on the HR diagram. Dots are the hot HdC stars.

(5) S-stars. It is possible to say roughly that the S-stars are M-stars with strong $\mathrm{Zi} \mathrm{O}$ bands.

(6) C-stars. These are carbon stars, their spectra have not TiO-bands, but $\mathrm{C}_{2}$, $\mathrm{CN}$ bands.

It is necessarly to point out that the S-characteristics may be not so strong. In that case we give to the star the class SM or MS. So that there are some intermediate stars between $\mathrm{M}$ - and S-type stars. There is a small group of C-stars, which show S-characteristics. These are SC and CS stars. All this means that some groups mentioned above are connected with other groups.

(7) HdC-stars which are hydrogen deficient carbon stars. These stars have rather weak hydrogen lines and remarkable features of carbon both atomic and molecular. This is an inhomogenous group, which contains stars with different characteristics. In particular their temperatures vary in a wide range. There is a subgroup of variable stars. It is so-called R CrB stars, which show suddenly great decreases of their brightness.

There are a few stars, which are difficult to classify into some groups mentioned above. We will not discuss them here. In this report we will concentrate our attention on the typical peculiarities of chemical composition of stars.

Let us start by discussing the normal $\mathbf{K}-\mathbf{M}$ giants. We called them 'normal' for two reasons. First, the number of these stars is above $80 \%$ of yellow and red giants. Second, the abundance pecularities of these stars are smaller than that of other group.

We will concentrate our attention on the following abundance ratios: 
(1) The relative abundances of the light elements.

(2) The ratio of carbon isotopes ${ }^{12} \mathrm{C} /{ }^{13} \mathrm{C}$.

(3) The relative abundances of the metals of the iron group in comparison with hydrogen, $\mathrm{M} / \mathrm{H}$.

(4) The relative abundances of the elements, which were produced by $s$-processes. The ratio $[\mathrm{Ti} / \mathrm{Zr}]$ characterizes that.

(5) The presence of short-living elements in the atmospheres such as technetium.

(6) The abundances of lithium.

For M-stars we have now not so well established data, but there are estimates of abundances of some elements.

The relative abundance of different elements in the atmospheres of normal K-stars do not differ significantly from that in the solar atmosphere (Conti et al., 1967). There is a strong difference of the carbon isotope ratio only. The $\mathrm{K}$-stars have the ratio ${ }^{12} \mathrm{C} /{ }^{13} \mathrm{C}$ less than 10 , while the solar value is about 90 (Greene, 1969).

The chemical compositions of $\mathbf{M}$ stars is not known in detail yet. Spinrad and Vardya (1966) have shown that the ratio $\mathrm{O} / \mathrm{C} \approx 1.05$ is less than the solar value 1.7 . The nitrogen abundance is not the same for all stars. $\alpha$ Her and $o$ Cet have the normal ratio $\mathrm{N} / \mathrm{H}$, but $\alpha$ Ori and $\mathrm{R}$ Leo have this ratio ten times more. The ratio ${ }^{12} \mathrm{C} /{ }^{13} \mathrm{C}$ is less than 8 for the $M$ stars investigated (Gaball et al., 1972).

Peery (1971) has found technetium lines in the spectra of two M-type miras $o$ Cet and R Hya. According to Merchant (1967) M stars have a lithium abundance much less than the Sun.

The next group is the stars with strong cyanogen molecular bands. Greene (1969) has shown that the carbon and the nitrogen have little enhancement. The abundance of nitrogen for $\mathrm{CN}$ stars seems to be very near to that for normal stars (Schmitt, 1969). The ratio ${ }^{12} \mathrm{C} /{ }^{13} \mathrm{C}$ is equal 10 for $\alpha$ Ser. The metals show a little deficiency about -0.2 dex.

Then we consider a group of BalI-stars. Here we will follow mainly Warner (1965). On the Figure 2 we can see the differences between stellar abundance ratios and that of the Sun. The main features are following (a) large abundance of lithium, (b) large enhancement of elements heavier than $\mathrm{Sr}$, (c) small overabundance of $\mathrm{Eu}$ and $\mathrm{Yb}$; that is $r$-process elements.

In general, metals are over-abundant by a factor 1.5 in comparison with hydrogen. The ${ }^{12} \mathrm{C} /{ }^{13} \mathrm{C}$ ratio in the BaII stars is probably more than 20 . No Tc-lines were observed in any BaII stars. $\mathrm{CH}$-stars are very similar to BaII stars. These stars have strong $\mathrm{CH}$ bands; Figure 3 shows us the differential abundance of two $\mathrm{CH}$ stars according to results of analysis by Wallerstein and Greenstein (1964).

We may see that the peculiarities of the abundance ratio $\log (\mathrm{M} / \mathrm{Fe})$ are very similar to those which we observed in Ba-stars. The heavy elements, which were produced by the $s$-process are overabundant. The carbon is overabundant also. The metals are deficient in respect to hydrogen by a factor 10 . The ${ }^{12} \mathrm{C} /{ }^{13} \mathrm{C}$ ratio is close to 50. No technetium was observed in $\mathrm{CH}$-stars. The strength of the $\mathrm{CH}$ band in comparison with atomic lines is explained by the high abundance of hydrogen. 


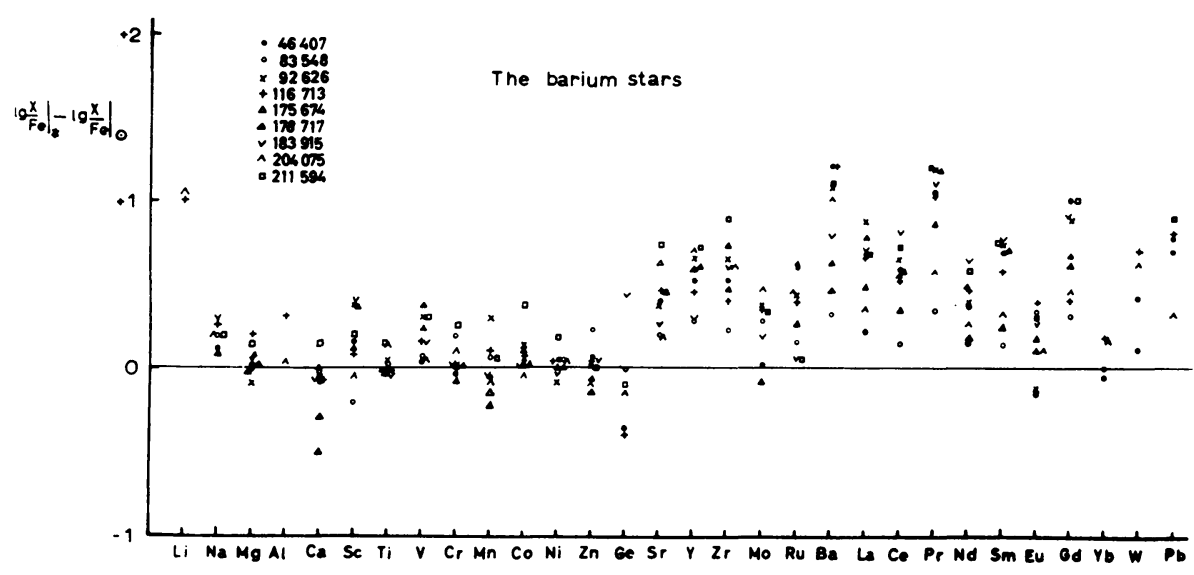

Fig. 2. The relative abundances in the atmospheres of some Ba-stars.

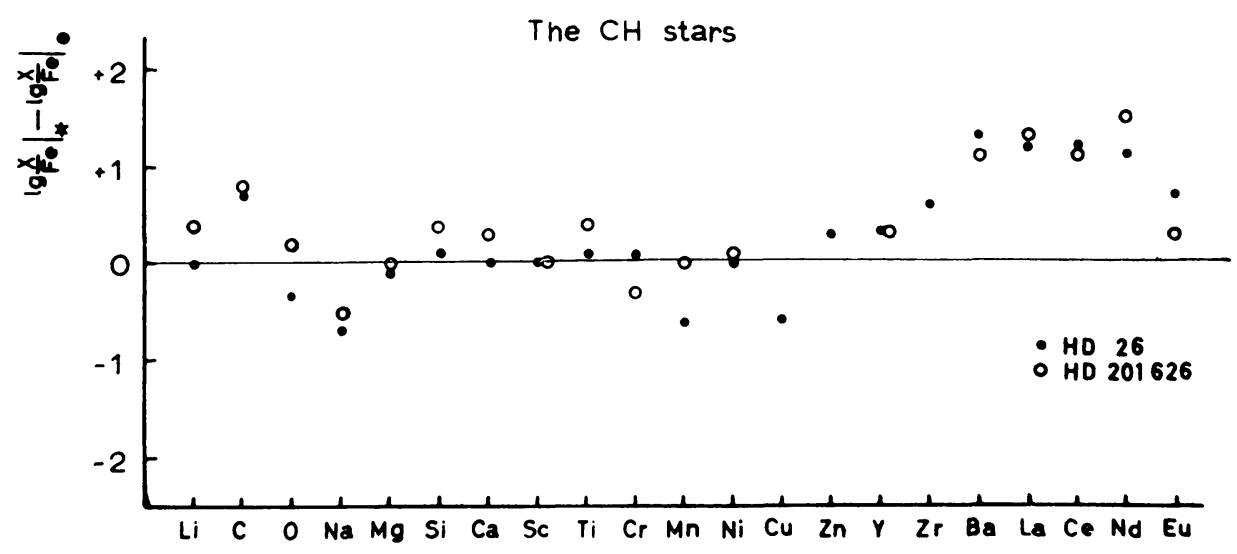

Fig. 3. The relative abundances in the atmospheres of two CH-stars.

The $\mathrm{CH}$ stars have large space velocities. Then we can consider them as Ba-stars of the Population II.

Let us consider now $\mathrm{S}$ stars. First of all it is necessary to point out that the accuracy of the determination of chemical composition of these stars is much less than that of K-type stars or earlier.

But nevertheless we can draw some conclusions about their chemical composition. The Figure 4 shows the chemical compositions of $S$ and $C$ stars according to Tsuji (1962, 1971) and Hirai (1969). This figure shows that the heavy elements are overabundant here more than in Ba-stars.

Then we can see that $\mathrm{C}$ stars contain more heavy elements than $\mathrm{S}$ stars. There are some differences of abundances of $r$ - and $s$-process elements: the $s$-process elements such as barium are over-abundant much more than $r$-process elements as europium. The relative abundance of oxygen group elements in the $S$ stars do not differ from that 


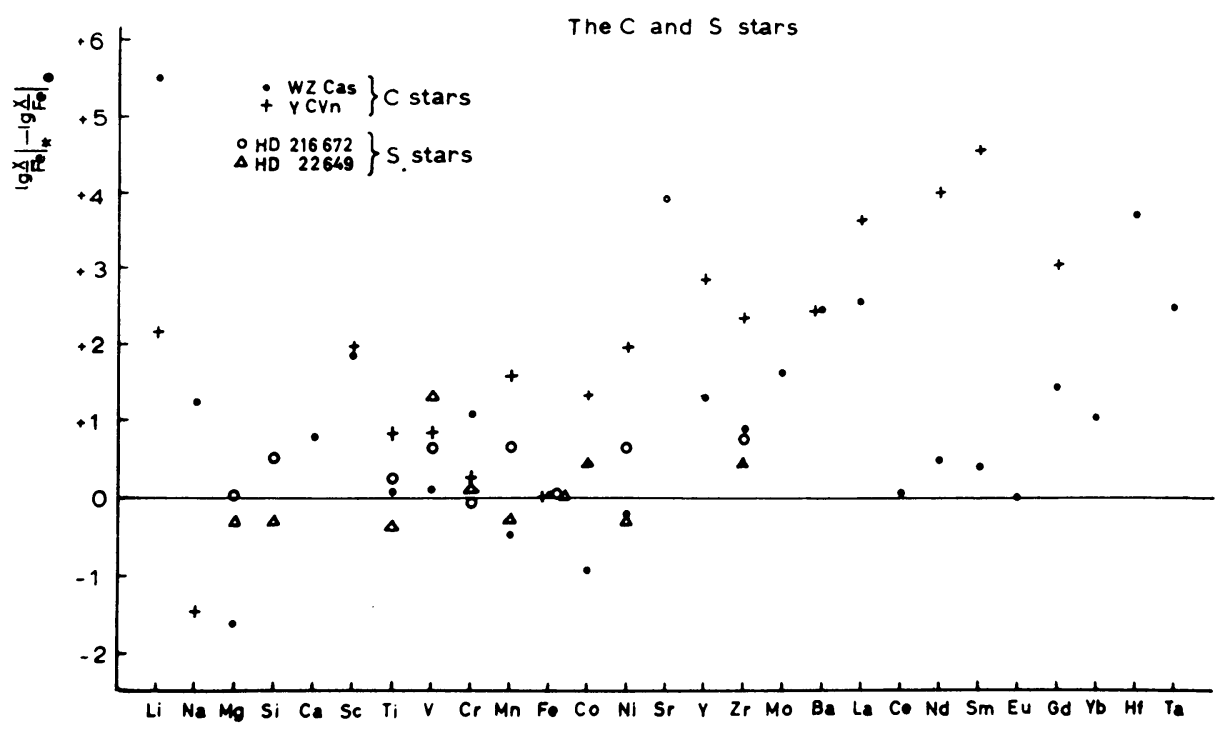

Fig. 4. The relative abundances in the atmospheres of $\mathrm{C}$ and $\mathrm{S}$ stars.

of the Sun by more than a factor 2. R And has ${ }^{12} \mathrm{C} /{ }^{13} \mathrm{C}=6$ (Tsuju, 1971). According to Peery (1971) the lines of Tc are present in spectra of variable S-type stars and absent in spectra of nonvariable stars. This is true for all stars which have been checked for Tc.

Davis (1971) has suspected the presence of the lines of prometheum in the spectra of two S-stars V Cnc and T Sgr. The abundance of lithium varies in a wide range.

The main feature of $\mathrm{C}$ stars is a high abundance of carbon. The ratio $\mathrm{O} / \mathrm{C}$ is less than unity. The carbon and oxygen form the tightly bound molecule $\mathrm{CO}$. Then the excess of carbon forms such molecules as $\mathrm{CH}, \mathrm{CN}$ and $\mathrm{C}_{2}$. But at the same time there is not enough oxygen in order to form oxides such as $\mathrm{TiO}$ and $\mathrm{ZrO}$, which are typical for M-type stars. The ratio $\mathrm{O} / \mathrm{C}$ in $\mathrm{C}$ stars is 20 times less than that in the Sun. In most cases the ratio ${ }^{12} \mathrm{C} /{ }^{13} \mathrm{C}$ is near to the equilibrium value in the $\mathrm{CN}$ cycle (4.6). But a few stars have the ratio ${ }^{12} \mathrm{C} /{ }^{13} \mathrm{C}$ near 20 (Querci and Querci, 1970). 6 stars from 8 investigated have Tc lines (Peery, 1971).

Lithium abundances vary in a very wide range, over 6 orders of magnitude (TorresPeimbert and Wallerstein, 1966). Some stars have lithium abundances 4 orders of magnitude more than that of the Sun. Usually these stars have also a high abundance of ${ }^{13} \mathrm{C}$. The ratio ${ }^{12} \mathrm{C} /{ }^{13} \mathrm{C} \approx 3$ (Gordon, 1971).

One star has been investigated by Catchpole and Feast (1971) for lithium isotopes. It was found that the whole lithium is ${ }^{7} \mathrm{Li}$.

Let us go to the left from the red giant branch and discuss $\mathrm{R} C r B$ stars. Only two stars were analysed. They are R CrB itself (Searle, 1961) and RY Sgr (Danziger, 1965). The results are shown in Figure 5 . We can see the large deficiency of hydrogen and oxygen. The helium, lithium, carbon and nitrogen are overabundant. Approximately the same position on the HR diagram is occupied by the non-variable stars, 


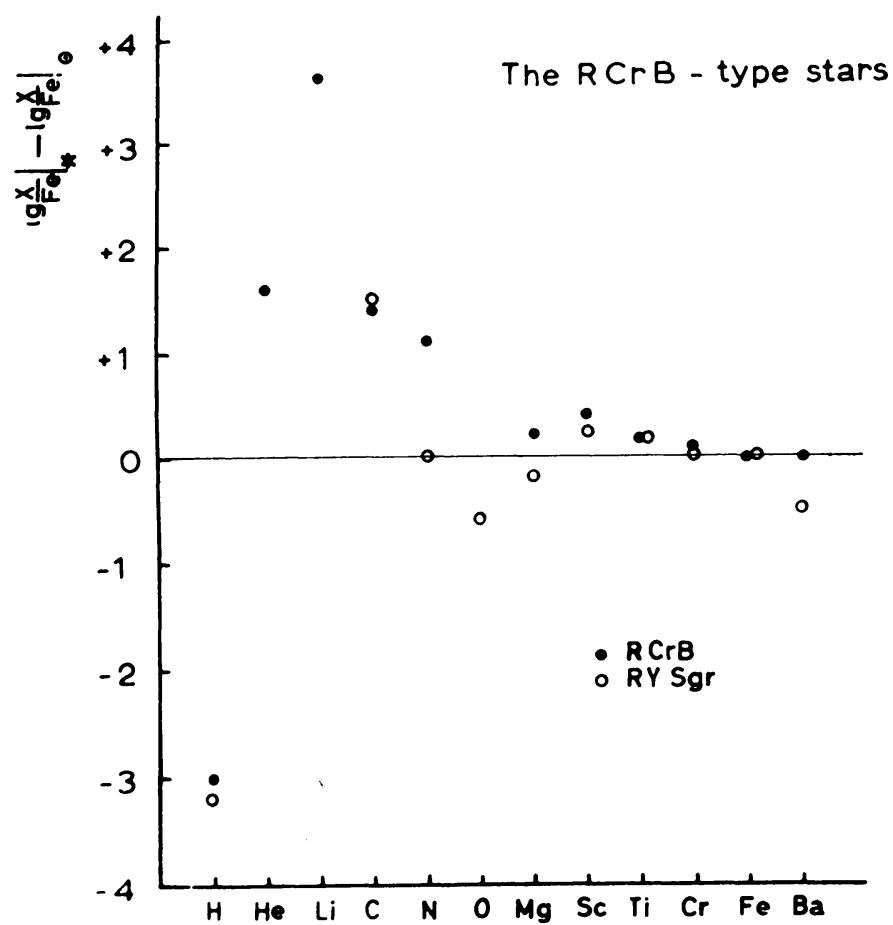

Fig. 5. The relative abundances in the atmospheres of $\mathrm{R} \mathrm{CrB}$ type stars.

The cool non-variable HdC stars

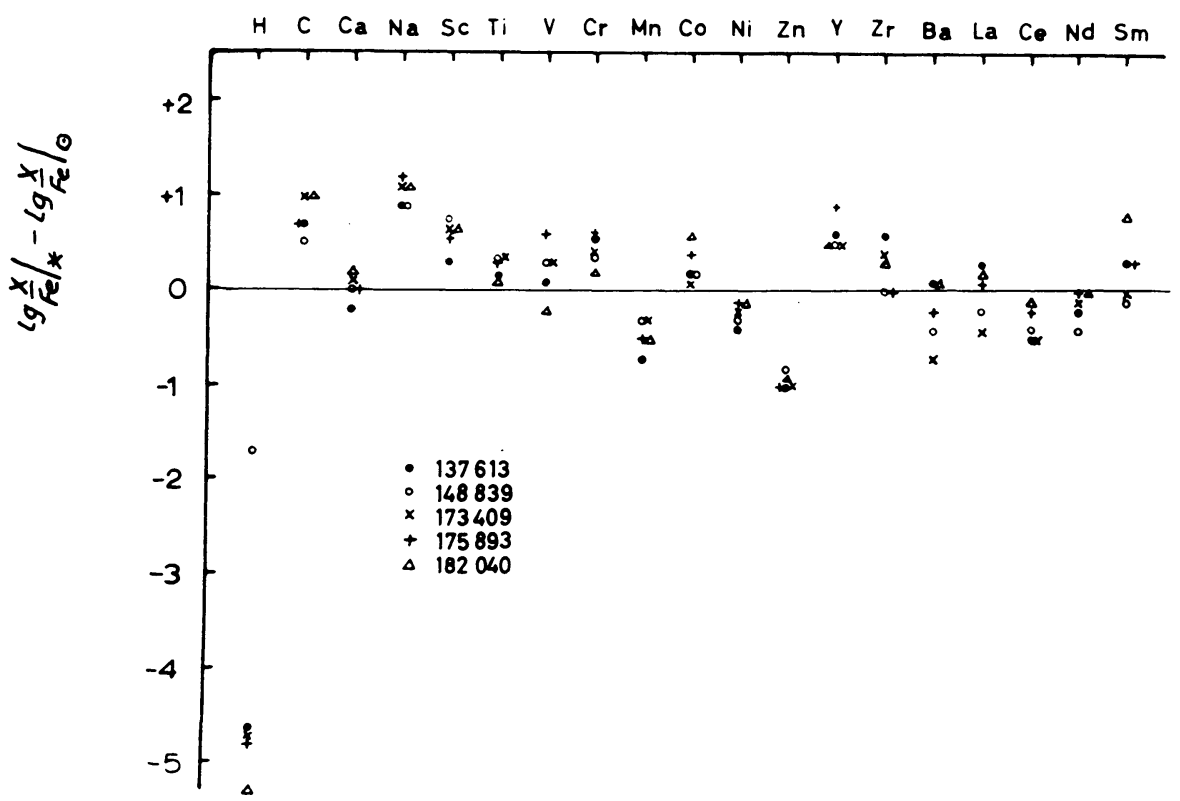

Fig. 6. The relative abundances in the atmospheres of cool HdC stars. 
The not HdC stars

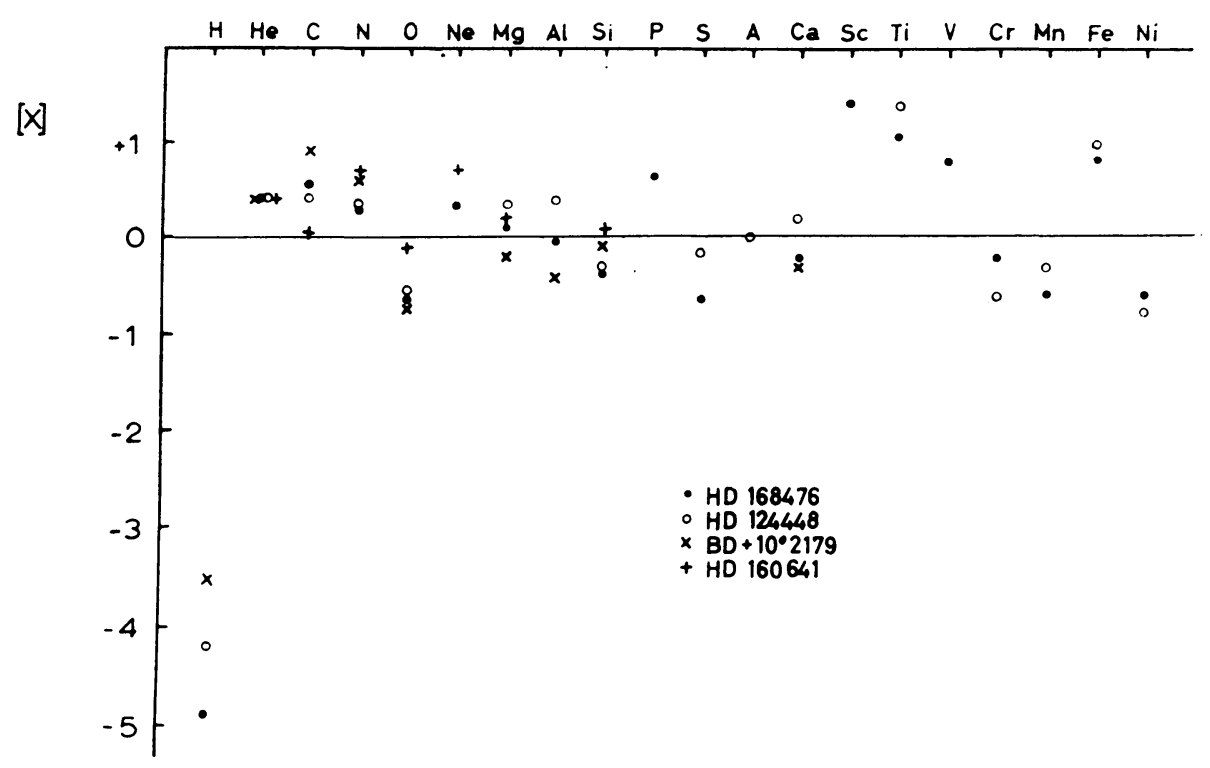

Fig. 7. The relative abundances in the atmospheres of hot HdC stars.

which have chemical composition similar to R CrB-type stars. These are so-called HdC-stars. Figure 6 represent the results of an analysis by Warner (1967). The main features of the abundances are the deficiency of hydrogen and the excess of carbon.

There are a few stars, which are located to the left of the cool HdC-stars and have the same situation with abundances of hydrogen and carbon. The Figure 7 shows the chemical compositions of some of these stars (Hill, 1965).

We see, that the hydrogen is strongly underabundant. The $\mathrm{He}, \mathrm{C}, \mathrm{N}$, is little overabundant and $\mathrm{O}$ little underabundant. We have a large scatter for the elements of iron group. This perhaps is a result of weakness of lines of those elements.

Table I gives a summary of abundance differences of some groups of late type.

TABLE I

\begin{tabular}{lcccccccc}
\hline & {$[\mathrm{C} / \mathrm{H}]$} & {$[\mathrm{N} / \mathrm{C}]$} & {$[\mathrm{O} / \mathrm{C}]$} & ${ }^{12} \mathrm{C} /{ }^{13} \mathrm{C}$ & {$[\mathrm{M} / \mathrm{H}]$} & {$[\mathrm{Ti} / \mathrm{Zr}]$} & $\mathrm{Tc}$ & {$[\mathrm{Li}]$} \\
\hline Normal M & 0 & +0.3 & -0.2 & $<8$ & 0.0 & -0.1 & yes & -2.0 to +1.0 \\
CN & +0.2 & +0.2 & 0 & $<10$ & -0.2 & 0.0 & & \\
CH & 0 & & & 50 & -1.1 & -0.5 & no & 0 \\
BaII & +0.6 & & & $>20$ & +0.2 & -0.7 & no & +1.0 \\
S & 0 & +0.3 & -0.3 & 6 & & -0.7 & yes & -1.5 to +3.0 \\
C & & -0.1 & -1.3 & $4-20$ & & -3.0 & yes & -2.5 to +4.3 \\
HdC & +5 & 0 & -1.3 & & & -0.5 & & +3.5 \\
R CBr & +4 & -0.3 & -2.0 & $>50$ & +3.0 & & &
\end{tabular}




\section{References}

Arp, H. C.: 1962, Astrophys. J. 136, 66.

Catchpole, R. M. and Feast, M. W.: 1971, Monthly Notices Roy. Astron. Soc. 154, 197.

Conti, P., Greenstein, J. L., Spinrad, H., Wallerstein, G., and Vardya, M. S.: 1967, Astrophys. J. 148, 105.

Danziger, I. J.: 1965, Monthly Notices Roy. Astron. Scc. 130, 199.

Davis, D. N.: 1971, Astrophys. J. 167, 327.

Eggen, O. J. and Sandage, A.: 1969, Astrophys. J. 158, 669.

Gaball, T. R., Wallman, E. R., and Rank, D. M.: 1972, Astrophys. J. 177, L27.

Greene, T. F.: 1969, Astrophys. J. 157, 737.

Gordon, C. P.: 1971, Publ. Astron. Soc. Pacific 83, 667.

Hill, P. W.: 1965, Monthly Notices Roy. Astron. Soc. 129, 137.

Hirai, M.: 1969, Publ. Astron. Soc. Japan 21, 91.

Merchant, A. E.: 1967, Astrophys. J. 147, 587.

Pagel, B. E. J.: 1970, Quart. J. Roy. Astron. Soc. 11, 172.

Peery, B. F. J.: 1971, Astrophys. J. 163, L1.

Querci, M. and Querci, P.: 1970, Astron. Astrophys. 9, 1.

Schmitt, J. L.: 1969, Publ. Astron. Soc. Pacific 81, 657.

Searle, L.: 1961, Astrophys. J. 133, 531.

Spinrad, H. and Vardya, M. S.: 1966, Astrophys. J. 146, 399.

Torres-Peimbert, S. and Wallerstein, G.: 1966, Astrophys. J. 146, 724.

Tsuji, T.: 1962, Publ. Astron. Soc. Japan 14, 222.

Tsuji, T.: 1971, Publ. Astron. Soc. Japan 23, 275.

Wallerstein, G. and Greenstein, J. L.: 1964, Astrophys. J. 139, 1163.

Warner, B.: 1965, Monthly Notices Roy. Astron. Soc. 129, 263.

Warner, B.: 1967, Monthly Notices Roy. Astron. Soc. 137, 119. 